Pincus's thesis that the methods of Nature can be improved on by the intelligent application of biological discovery.

R. G. EDWARDS

\section{COMPARATIVE INVERTEBRATE NEUROLOGY}

\section{Structure and Function in the Nervous Systems of Invertebrates}

By Prof. Theodore Holmes Bullock and Dr. G. Adrian Horridge. With ehapters by H. A. Bern, I. R. Hagadorn and J. E. Smith. Vol. 1: Pp. xx +1-798. Vol. 2: Pp. viii +799-1719. (San Francisco and London: W. H. Freeman and Company, 1965.) 540s. per set.

NVERTEBRATE neurophysiologists have been com1 pelled to work with a large, scattered, and unwieldy literature; Hanström's Vergleichende Anatomie des Ner. vensystems der wirbellosen Tiere appeared in 1928, and there has been no comprehensive account of invertebrate neurophysiology. Tho authors of Structure and Function in the Nervous Systems of Invertebrates have attempted to survey the present state of both these fields of endeavour. While I am still concussed by the magnitude of the enterprise, its scope may be indicated by a brief summary of the contents.

Part 1 (429 pp.) establishes the general mechanisms and principles governing the operation of nervous systems, and includes sections on micro-anatomy, excitability and conduction, transmission and integration, and a contribution on neurosecretion by H. A. Bern and I. R. Hagadorn. Parts 2-5 describe the special features of the groups and deal respectively with the lower Phyla (365 pp.), the Arthropoda (469 pp.), the Mollusca (242 pp.), and the Deuterostomes (70 pp.).

It must be said at the outset that the bibliography alone makes these books indispensable. Throughout, they are lavishly illustrated with photomicrographs, electron micrographs and excellent line-drawings. In addition to a careful use of headings and summaries, bold type is deployed in the text in two ways: to emphasize key words which indicate the topic under discussion, and to underline phrases which the authors feol to be of particular importance. This device, in practice, works well enough, although I find it visually rather irritating; occasionally, a phrase stands out from its surroundings with merely eccentric effect: "lower phyla are on an equal plane with the nervously more advanced animals", for example (Volume 1, p. 271). However, the ease with which a particular topic can be run down more than compensates for these disadvantages. In general, the text is lucid, although there are some startling lapses, which sometimes seem to result from tco strenuous an attempt at concision. Thus, on p. 16 of Volume 1 a paragraph, clearly intended to stand by itself, reads: "Correlated with cell-types is the histological architecture of nervous tissue. Whenever it occupies the superficial, basepithelial position characteristic of the simplest systems (coelenterate, echinoderm, enteropneust, the epidermal plexus of nemerteans, even the brain and cord of some simple acoel flatworms, terebellids and limicolous oligochaetes), the diagnostic tissue, a stratum of nearly cell-free nervefibres, lies deep in the epithelium, just external to the basement membrane, if present, and to muscle-fibres. It is visible even in sections stained by common unselective dyes as a layer of punctate texture but little structure, unless locally there are too few fibres to form a distinct layer." The paragraph which precedes this discusses the classification of neurones in terms of the differentiation of their processes, and it is followed by a paragraph on the organization of ganglia. It is not immediately apparent that the section I have quoted is intended to define the characteristics of a basepithelial nervous system, for the subject is neither mentioned by name, nor given the benefit of bold type.

In fact, the merits of these volumes are distributed in a way which clearly reflects the interests of the two authors; both are neurophysiologists of distinction, and the sections which display our present understanding of neural function are admirably comprehensive and well organized. They can be used as a quick source of information and read for pleasure, and to combine both roles in one text is a remarkable achievement. Nevertheless, only a small proportion of invertebrate nervous systems has been investigated physiologically, and many chapters are, in the nature of things, almost solely concerned with structure. Whenever an equivocal anatomical concept or finding affects a physiological hypothesis (for instance, the neurone doctrine, or controversies opposing continuity versus contiguity at synapses), we are given a scholarly and historical summary of the several points of view. But it does not help the neuroanatomist to be told, somewhat baldly, that dendrites can be defined as "those processes of neurones specialised as though to act as receptor regions for the soma", for he is more concerned to find labels which will enable him to categorize the units which he sees in his preparations, and the physiological function of which is probably quite unknown. He should, perhaps, be warned against the rising dangers of applying rigid terminologies with functional implications to structures which, in invertebrates, are often physiologically ambiguous.

But the pitfalls of neuroanatomical interpretation go far beyond matters of definition: they are implicit in the very nature of the histological methods which are available. Where so much depends on the limitations of individual methods of metallic impregnation, and on the difficulties of combining the different kinds of information which they provide, it is disappointing to find only a brief discussion of these techniques and of their application in practice. This is particularly serious in the case of such structures as the optic lobes of insects, for the amount of reliance that can be placed on the early accounts by Cajal and Sanchez and by Zawarzin requires some knowledge of tho vagaries of impregnations of the Golgi type and of methylene blue staining respectively. The authors do not always tell us how the information which they present was obtained.

Obviously, a single reviewer is not competent to assess all the chapters. Those dealing with the Insecta manage to cover a vast range of material, but it is not always well organized. There appear to be some inconsistencies in the nomenclature of the optic tracts, and here a table giving the usages of the various sources would have helped. There is one curious omission: despite the importance of the corpora pedunculata, and a good account of recent work on their physiology and fine anatomy, the beautiful and accurate figures from Apis which Kenyon published in 1896 are nowhere represented. The exact topography of the intrinsic neurones of these structures, and of the afferent and efferent endings which lie within them, are crucial to hypotheses about the ways in which they may work, and only in this early study have they been displayed by selective silver methods.

These comments may seem ungenerous. Against them may be set the difficulties inherent in this monstrous labour of love, and the improbability of anyone attempting it again. Few comparative physiologists could have achieved the very real morits of these volumes, or have scoured the literature with the diligence and thoroughness which are everywhere evident. A physiologist who works in any one of the fields covered by this treatise is naturally inclined to look for the kind of critical evaluation of the literature which he would hope to find in a specialized review. Such hopes are extravagant; wo should be grateful for what the authors have given us.

A. D. Brest 